\title{
Le mythe de la guêpe maçonne
}

(ou les pièges de l'observation en biologie)

Valdiodio Ndiaye et Pierre Clément

\section{CpenEdition}

Journals

Édition électronique

URL : http://journals.openedition.org/trema/2044

DOI : $10.4000 /$ trema.2044

ISSN : 2107-0997

Éditeur

Faculté d'Éducation de l'université de Montpellier

Édition imprimée

Date de publication : 1 septembre 1996

Pagination : 105-110

ISSN : 1167-315X

Référence électronique

Valdiodio Ndiaye et Pierre Clément, «Le mythe de la guêpe maçonne », Tréma [En ligne], 9-10 | 1996, mis en ligne le 18 septembre 2013, consulté le 02 mai 2019. URL : http://journals.openedition.org/ trema/2044 ; DOI : 10.4000/trema.2044

Ce document a été généré automatiquement le 2 mai 2019.

Trema 


\title{
Le mythe de la guêpe maçonne
}

\author{
(ou les pièges de l'observation en biologie)
}

\section{Valdiodio Ndiaye et Pierre Clément}

\section{Introduction}

1 À Dakar (Sénégal), un enseignement de Biologie, destiné à de futurs professeurs de Sciences Naturelles de lycées et collèges, a été émaillé par un débat sur la guêpe maçonne, qui est ensuite devenu une référence exemplaire au point d'être utilisée assez systématiquement dans les enseignements suivants de Biologie. Exemplaire quant à la force avec laquelle le contexte culturel et social des apprenants façonne leurs conceptions (le terme de conceptions est utilisé dans le sens précisé par Clément, 1994, et généralement admis dans la communauté des didacticiens de la Biologie). Exemplaire quant à la dépendance de toute observation par rapport aux conceptions de l'observateur.

Figure 1

Une guêpe maçonne femelle, la guêpe des sables Amnophila adriansei (Hyménoptère, Sphécidés). Elle traîne vers son nid une chenille de Géométride qu'elle vient de paralyser. L'entrée de son nid (à gauche) est fermée avec de petits cailloux. Remarquer la taille effectivement très fine et très longue de cette amnophile. (document modifié d'après Grzimek B., 1975).

GRZIMEK B., " Les hyménoptères ", in Le monde animal, Zurich, Suisse, Éd. Stauffacher S.A., 1975.

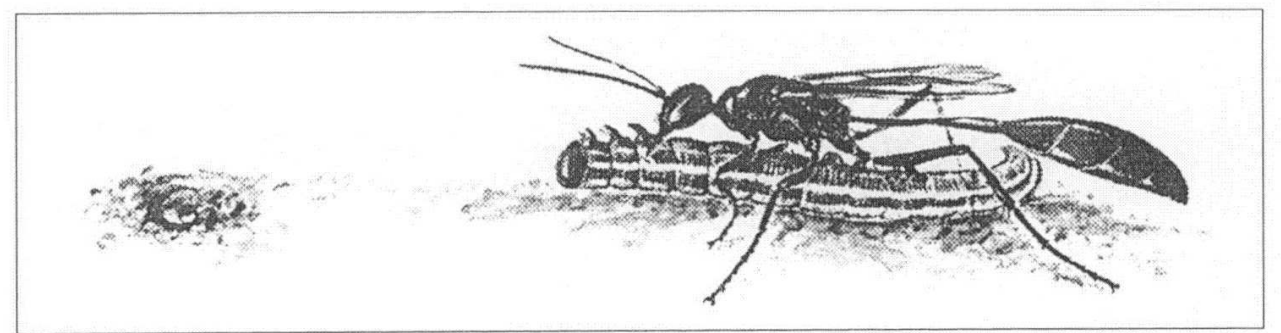

2 Le mythe de la guêpe maçonne est familier à une large majorité de la société sénégalaise, où il est en permanence renforcé par des observations simples, sortes d'évidences qui 
logiquement le "prouvent", tout en défiant les interprétations scientifiques des mêmes phénomènes. Faire un détour par cette logique inhabituelle à nos yeux d'occidentaux nous a semblé intéressant, pour lui comparer notre logique scientifique et juger jusqu'à quel point elle en est différente ou non. Nos observations scientifiques, et celles que nous attendons de nos élèves ou étudiants, ne sont-elles pas influencées par nos croyances et nos attentes: et si celles-ci sont "scientifiques", cela justifie-t-il des raccourcis et approximations qui nous semblent insupportables quand ils sont empruntés par ceux qui fonctionnent à partir d'autres croyances?

\section{Un mythe où se mêlent observations et croyances}

Voici une transcription écrite approximative de ce mythe oral.

«Au tout début, Dieu, poursuivant son cuvre créatrice, demanda à l'assistance de se détourner ou de se bander les yeux pour ne pas pénétrer son secret sous peine de sanction. Tous se soumirent à la consigne, sauf la guêpe maçonne qui, se cachant les yeux avec ses pattes, laissa un espace pour voir comment s'y prenait Dieu pour créer la vie. Elle réussit ainsi à déflorer le secret divin. Mais Dieu s'en aperçut. Pour la punir de sa désobéissance, il lui serra le corps par la taille, si fort que celle-ci n'est plus apte, du fait de son rétrécissement à abriter et surtout à laisser passer une progéniture.

Ainsi, la guêpe est condamnée à ne jamais connaître la joie d'enfanter, de donner une descendance par ses entrailles. Mais comme elle a acquis le savoir-faire divin, elle construit son nid dans lequel, à l'abri des regards indiscrets, elle crée la vie, elle recrée de nouvelles guêpes maçonnes, à partir de larves vermiformes d'autres insectes qu'elle ramasse au dehors et introduit dans son nid. C'est ainsi qu'existent encore aujourd'hui des guêpes maçonnes. ”

Ce mythe du mode de reproduction de la guêpe maçonne est raconté à la plupart des enfants sénégalais, dans leur milieu familial, avant leur scolarisation.

Quels sont les fondements du mythe?

- Des observations/interprétations vérifient ce que dit le mythe. Tous les enfants sénégalais qui ont écouté ce récit vérifient, dans leurs observations de tous les jours, la concordance entre ce qu'ils voient et ce qui leur a été rapporté. La conformité entre le mythe et l'expérience quotidienne est satisfaisante. La taille de la guêpe est étonnamment longue et fine, comme une aiguille. La guêpe ramène au nid un vers, une larve d'insecte souvent plus grosse qu'elle, après l'avoir paralysée (figures $1 \& 2$ ). En détruisant le nid, l'enfant y vérifie la présence de ces vers/proies (figure 3), ou observe la présence d'une coque un peu rigide qu'il interprète comme la transformation progressive de ces vers/proies en guêpes.

- L'interprétation est cohérente avec des croyances préalables. L'interprétation des observations faites par l'enfant sénégalais sur ces guêpes maçonnes et sur leurs nids, est en parfaite cohérence avec son contexte culturel mythico-religieux. Il est en effet élevé dans une croyance en la création divine, et dans la possibilité d'une transmutation, c'est-à-dire d'une transformation spontanée, actuelle, de certains êtres vivants en d'autres êtres vivants d'espèces différentes. Ce modèle suffit pour conforter ses observations et interprétations spontanées sur la guêpe maçonne, et donc le satisfait entièrement.

- L'enseignement scolaire en biologie porte sur d'autres espèces que la guêpe maçonne. Pourquoi ne pas en même temps croire aux métamorphoses complètes du hanneton ou de l'abeille, enseignées par le professeur (souvent de façon magistrale, sans observations probantes), et continuer à croire en l'exception de la guêpe maçonne, enseignée par le mythe ? 


\section{Une explication alternative (scientifique) : au-delà des croyances et observations}

5 Le professeur de Sciences Naturelles sait que la guêpe maçonne, comme tous les hyménoptères, est un insecte à métamorphoses complètes (holométabole) : à partir des œufs déposés dans le nid par la guêpe maçonne - mère, des larves vermiformes naissent puis grandissent par mues successives jusqu'à se transformer en nymphes pour la métamorphose. À la fin de celle-ci, la peau de chaque nymphe se fend et il en émerge une guêpe adulte qui prend son envol. Les larves de guêpes maçonnes sont très différentes des adultes. Enfermées dans le nid de terre construit par leurs parents, ces larves sont nourries grâce à des vers (larves d'autres insectes, chenilles de papillons dans les exemples illustrés par les figures $1 \& 2$ ) que les adultes ramassent hors du nid, en les paralysant et les transportant entiers dans le nid (ce qui est effectivement assez spectaculaire, car les vers transportés sont souvent bien plus volumineux que la guêpe elle-même). En résumé, nous, enseignants biologistes, n'avons aujourd'hui aucun problème à bien différencier la transformation de ces vers en nourriture des larves de guêpes, de la transformation des œufs en larves puis en nymphes puis en adultes de guêpe maçonne (métamorphose).

\section{Figure 2}

Une guêpe maçonne, l'Eumène (Hyménoptère, Vespidés), transporte en volant une chenille paralysée de Bombycidé vers son nid d'argile en forme d'urne, (document modifié d'après Grzimek B., 1975).

GRZIMEK B., « Les hyménoptères », in Le monde animal, Zurich, Éd. Stauffacher S.A., 1975.

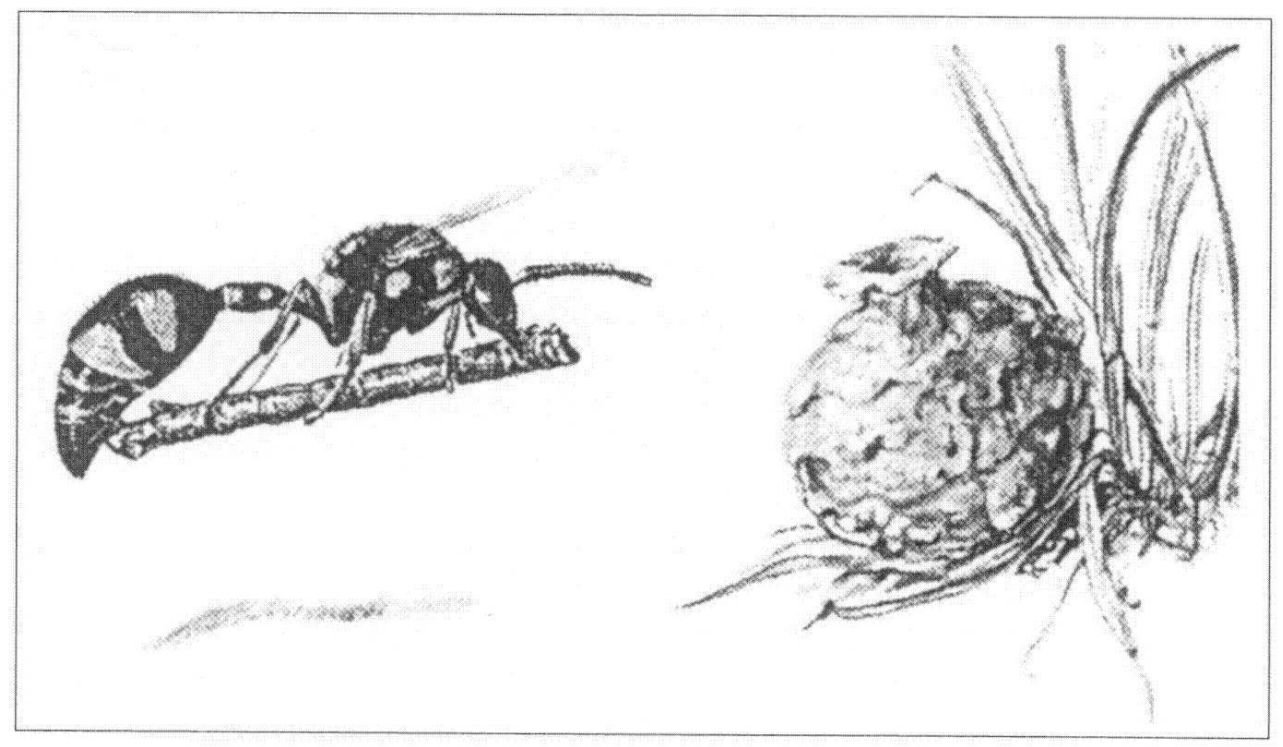

6 Les enseignants scientifiques que nous sommes adhérons sans hésitation à ces interprétations scientifiques. Pourtant, qui d'entre nous a observé des larves et des nymphes de guêpes maçonnes? Il est possible de répondre sans grands risques de se tromper : très peu. Mais nous fonctionnons par analogie avec les larves et les nymphes que nous connaissons (vers à soie, abeilles, hannetons...), et avec la certitude que tous les lépidoptères, hyménoptères et coléoptères ont de telles métamorphoses complètes : il ne saurait y avoir d'exception pour la guêpe maçonne! Nous pouvons d'ailleurs le vérifier si 
nécessaire : c'est là la grande force de la science : mais c'est aussi un principe qui n'est pas aisé à mettre en œuvre. Et les auteurs de ce présent texte ont eux-mêmes délicatement ouvert les alvéoles de nids construits par les guêpes maçonnes pour y découvrir sans surprise des larves vermiformes ou des nymphes; mais, avouons-le, ces observations ponctuelles auraient été interprétées bien différemment par ceux qui auraient cru au mythe: pour les convaincre, il aurait fallu pouvoir observer directement les transformations d'œuf en larve, puis de larve en nymphe et enfin de nymphe en adulte ailé. Nous sommes persuadés que ce serait possible si nous nous en donnions les moyens: par exemple si nous arrivions à faire construire certaines alvéoles du nid de guêpe contre une vitre transparente mais placée à l'obscurité, pour observer en lumière infrarouge (avec des lunettes spéciales, ou avec une caméra sensible aux infrarouges) l'éclosion puis les mues jusqu'à la nymphose et l'émergence de l'adulte. Mais quel travail pour faire que des élèves sénégalais puissent eux aussi effectuer de telles observations! Bien sûr, il reste possible de filmer, puis de leur projeter le film : même si, de nos jours, et notamment depuis Jurassic Park, un film ne prouve plus grand chose, la réalisation d'un tel documentaire mériterait d'être entreprise.

7 La fonction de la taille de la guêpe maçonne est tout aussi difficile à démontrer. La microdissection à la loupe binoculaire serait une prouesse technique pour un chercheur spécialisé, et reste donc hors d'atteinte d'un élève dans un contexte scolaire habituel. Des coupes histologiques sériées de la taille et de l'abdomen sont effectivement des preuves de l'activité reproductrice habituelle de la guêpe maçonne, et de son organisation anatomique d'hyménoptère non exceptionnel. Mais les pré-requis pour interpréter de telles coupes sont nombreux, et souvent sous-estimés au lycée: notions élémentaires d'histologie et d'anatomie; capacité à reconstituer des structures 3D à partir de coupes 2D de ces structures.

Dans l'immédiat, l'enfant sénégalais ne peut s'appuyer, au mieux, que sur des observations morphologiques ou comportementales dont l'interprétation n'est pas évidente. Il reste tiraillé entre le discours de son enseignant scientifique, et le mythe qui l'a imprégné depuis son plus jeune âge. Et même s'il observe en classe les métamorphoses d'un vers à soie, il garde le droit de penser que ça n'est pas pareil pour la guêpe maçonne, comme le prouve la finesse de sa taille. Pour la guêpe maçonne, il n'a pas de raisons décisives pour privilégier l'interprétation proposée par l'enseignant et par de lointains traités scientifiques, à l'interprétation proposée par une tradition familiale et sociale encore bien vivante et qui lui permet tout aussi bien d'interpréter ce qu'il peut observer sur ces guêpes. 
Figure 3

Trois urnes d'argile fabriquées par une Eumène (voir figure 2). Celle de gauche est représentée en coupe axiale : elle contient plusieurs chenilles qui sont des provisions destinées à la larve qui va éclore de l'œuf, celui-ci étant suspendu au plafond de l'urne par un fil. (document modifié d'après Grzimek B., 1975).

GRZIMEK B., « Les hyménoptères », in Le monde animal, Zurich, Éd. Stauffacher S.A., 1975.

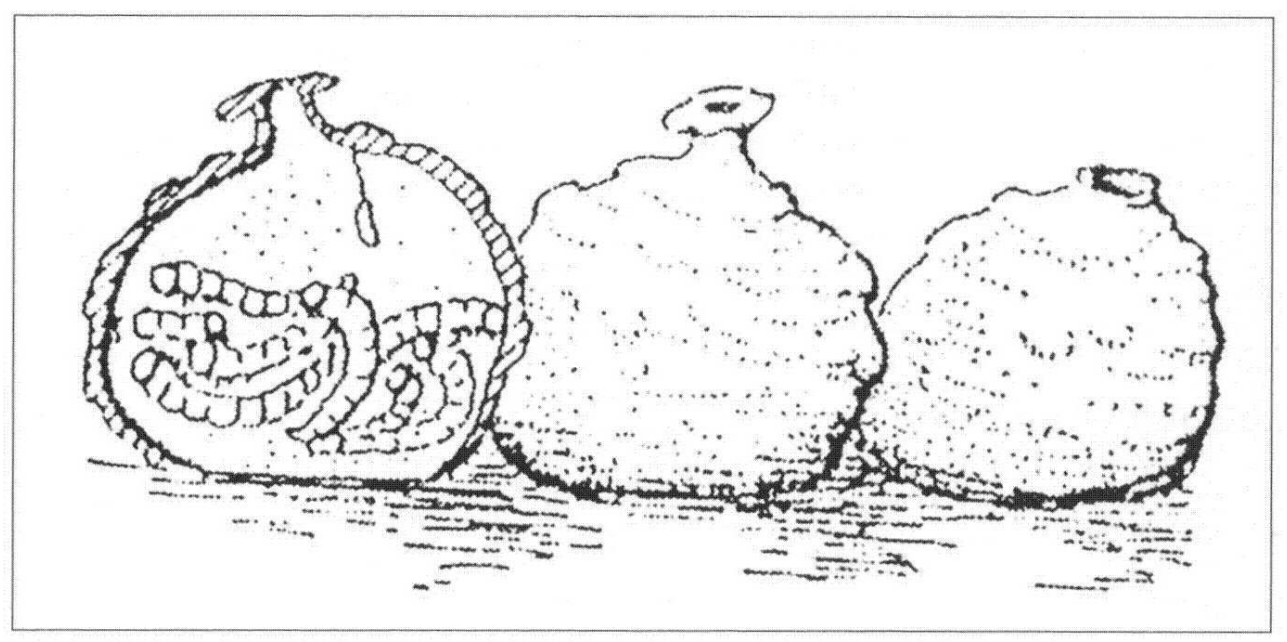

\section{Les leçons du mythe}

Ce mythe illustre les difficultés et les limites de l'observation en Biologie. Il conforte la plupart des philosophes des sciences dans leur critique de l'inductivisme naif (cf. par exemple Chalmers, 1987) : l'observation ne peut à elle seule être au point de départ des connaissances scientifiques.

Il nous rappelle que :

- nous observons avec notre culture et nos croyances ;

- nous observons ce que nous savons interpréter (en nous référant à un mythe ou aux connaissances scientifiques) ;

- nous nous limitons à ces observations qui sont généralement « suffisantes » en fonction de nos préoccupations. Dès que des observations confortent nos croyances/connaissances préalables, nous pensons les avoir prouvées! Alors nous généralisons, extrapolons souvent trop hâtivement.

Le mythe de la guêpe maçonne nous renvoie finalement au danger des connaissances premières du sens commun qui font obstacle à l'acquisition de connaissances scientifiques (Bachelard, 1938). Et l'obstacle est d'autant plus important qu'il s'enracine dans un mythe vivant.

11 Mais nous avons aussi vu un autre danger: celui de ne pas pouvoir distinguer l'enseignement des sciences de celui des mythes! Plus il est difficile (voire impossible) de prouver aux élèves ce qui est enseigné, plus les contenus scientifiques deviennent des dogmes à leurs yeux, et plus l'enseignement des sciences se confond avec celui des mythes et des religions... 


\section{BIBLIOGRAPHIE}

BACHELARD G., La formation de l'esprit scientifique, Paris, Éditions Vrin, 1938.

CHALMERS A.F., Qu'est-ce que la science ?, Paris, Éditions La Découverte, (pour la traduction française), 1987. - Traduit de l'anglais : "What is this Thing Called Science? An Assessment of the Nature and Status of Science and its Methods.", St Lucia, Australie, University of Queensland Press, 1976.

CLÉMENT P., « Représentations, conceptions, connaissances », in : Giordan A., Girault Y., Clément P., Conceptions et connaissances, Berne, Suisse, Éd. Peter Lang, 1994, p. 15-45,

GRZIMEK B., « Les hyménoptères », in Le monde animal, Zurich, Suisse, Éditions Stauffacher S.A., 1975.

\section{RÉSUMÉS}

Le mythe de la guêpe maçonne, et nos réactions à son égard, illustrent les contradictions logiques à l'origine des observations et interprétations et montrent ainsi les limites des observations et interprétations scientifiques, telles que celles qui sont proposées à nos élèves, et qui diffèrent si peu de ce que nous critiquons dans le fonctionnement du mythe. L'interaction entre nos représentations sociales et nos capacités et difficultés à observer et interpréter est soulignée.

The myth of the bricklayer wasp and our reactions to it, illustrate the logical contradictions which are at the basis of observations and interpretations, and thereby show the limits of scientific observations and interpretations, such as those which are proposed to our students, and which differ so very little from precisely that which we downgrade in the functioning of a myth. The interaction between society's way of representing reality and one's abilities and difficulties in observing is here interpreted and underscored.

\section{INDEX}

Mots-clés : biologie, observation, obstacle, représentation sociale

Keywords : biology, observation, obstacle, society's representation of

\section{AUTEURS}

\section{VALDIODIO NDIAYE}

ENS - université Cheikh Anta Diop, Dakar-Fann, Sénégal

\section{PIERRE CLÉMENT}

Didactique de la biologie, LIRDIMS, université Claude Bernard de Lyon 